1 High activity of an affinity-matured ACE2 decoy against Omicron SARS-CoV-2 and pre-

\title{
emergent coronaviruses
}

Running Title: ACE2 decoy maintains activity against emerging coronavirus variants

$5 \quad{ }^{1}$ Gene Therapy Program, Department of Medicine, Perelman School of Medicine, University of

$7 \quad$ * Corresponding author:

8 James M. Wilson, $\mathrm{MD}, \mathrm{PhD}$

9 Gene Therapy Program

10

Perelman School of Medicine

11 University of Pennsylvania

$12 \quad 125$ South $31^{\text {st }}$ Street, Suite 1200

13 Philadelphia, PA 19104, USA

14 Phone: 215-573-9020; Fax: 215-494-5444

15 E-mail: wilsonjm@upenn.edu 


\section{Abstract}

21 The viral genome of severe acute respiratory syndrome coronavirus 2 (SARS-CoV-2), and

22 particularly its cell-binding spike protein gene, has undergone rapid evolution during the

23 coronavirus disease 2019 (COVID-19) pandemic. Variants including Omicron now seriously

24 threaten the efficacy of therapeutic monoclonal antibodies and vaccines that target the spike

25 protein. Viral evolution over a much longer timescale has generated a wide range of genetically

26 distinct sarbecoviruses in animal populations, including the pandemic viruses SARS-CoV-2 and

27 SARS-CoV-1. The genetic diversity and widespread zoonotic potential of this group complicates

28 current attempts to develop drugs in preparation for the next sarbecovirus pandemic. Receptor-

29 based decoy inhibitors can target a wide range of viral strains with a common receptor and may

30 have intrinsic resistance to escape mutant generation and antigenic drift. We previously generated

31 an affinity-matured decoy inhibitor based on the receptor target of the SARS-CoV-2 spike protein,

32 angiotensin-converting enzyme 2 (ACE2), and deployed it in an adeno-associated viral vector

33 (rAAV) for intranasal delivery and passive prophylaxis against COVID-19. Here, we demonstrate

34 the exceptional binding and neutralizing potency of this ACE2 decoy against SARS-CoV-2

35 variants including Omicron, as well as binding to diverse ACE2-dependent coronaviruses. We also

36 discuss a strategy of decoy-based treatment and passive protection to mitigate the ongoing

37 COVID-19 pandemic and future airway virus threats. 


\section{Author Summary}

43 Viral sequences can change dramatically during pandemics lasting multiple years. Likewise,

44 evolution over centuries has generated genetically diverse virus families posing similar threats to

45 humans. This variation presents a challenge to drug development, in both the breadth of

46 achievable protection against related groups of viruses and the durability of therapeutic agents or

47 vaccines during extended outbreaks. This phenomenon has played out dramatically during the

48 coronavirus disease 2019 (COVID-19) pandemic. The highly divergent Omicron variant of

49 severe acute respiratory syndrome coronavirus 2 (SARS-CoV-2) has upended previous gains

50 won by vaccine and monoclonal antibody development. Moreover, ecological surveys have

51 increasingly revealed a broad class of SARS-CoV-2-like viruses in animals, each poised to cause

52 a future human pandemic. Here, we evaluate an alternative to antibody-based protection and

53 prevention - a decoy molecule based on the SARS-CoV-2 receptor. Our engineered decoy has

54 proven resistant to SARS-CoV-2 evolution during the ongoing COVID-19 pandemic and can

55 neutralize all variants of concern, including Omicron. Furthermore, the decoy binds tightly to a

56 broad class of sarbecoviruses related to pandemic SARS-CoV-2 and SARS-CoV-1, indicating

57 that receptor decoys offer advantages over monoclonal antibodies and may be deployed during

58 the COVID-19 pandemic and future coronavirus outbreaks to prevent and treat severe illness. 
65 Monoclonal antibody therapeutics with the ability to bind the spike protein of severe acute respiratory syndrome coronavirus 2 (SARS-CoV-2) and prevent cell entry have been critical

67 tools in managing the coronavirus disease 2019 (COVID-19) pandemic $(1,2)$. These drugs prevent hospitalizations when applied early in the course of infection (3) and can provide critical passive protection for vulnerable populations of immunocompromised patients who cannot mount a protective response to vaccines (4). However, monoclonals have proven particularly susceptible to SARS-CoV-2 evolution (5). This susceptibility may arise because the spike epitopes most sensitive to neutralization have been under intense selection as the virus has made gains in transmissibility and its ability to evade human immunity (6). Furthermore, evidence suggests that single monoclonals applied in a therapeutic setting can rapidly give rise to escape mutants (7-10). Together, these findings call into question the ability of the antibody platform to keep pace with the course of the COVID-19 pandemic or to be of use in future pandemics caused by other coronaviruses.

Receptor decoys may represent a mode of viral neutralization that is more resistant to continued

80 viral evolution and escape-mutant generation (11). SARS-CoV-2 evolution has occurred in a

81 way that retains tight binding to its primary cell entry receptor, angiotensin-converting enzyme 2

82 (ACE2) (12). We and others have developed affinity-matured, soluble ACE2 decoy molecules that potently neutralize SARS-CoV-2 (11, 13-18). Our soluble Fc-fused decoy, CDY14HL-Fc4,

84 contains six amino acid substitutions that improve the neutralization of CoV-2 variants by 300 -

85 fold versus un-engineered ACE2 and an active site nutation to ablate its endogenous angiotensin- 
86 cleaving activity. Furthermore, CDY14HL maintains tight binding or neutralizing activity for the

87 distantly related sarbecoviruses WIV1-CoV, and SARS-CoV-1 despite being engineered for

88 improved activity against SARS-CoV-2 (13). This property suggests that this decoy may be a

89 useful tool to combat future pandemics from currently pre-emergent, ACE2-dependent

90 coronaviruses.

91

92 Here, we evaluate the binding and neutralization activity of CDY14HL against a wide range of

93 emerging SARS-CoV-2 variants, including Omicron, and pre-emergent ACE2-dependent

94 coronaviruses. These studies suggest the broad utility of decoy-based viral entry inhibitors in

95 combating current and future coronavirus pandemics.

96

97

98

99

100

101

102

103

104 


\section{Results}

\subsection{CDY14HL maintains tight binding to diverse SARS-CoV-2 variants}

We set out to evaluate the ability of our engineered ACE2 decoy to neutralize emerging SARS-

CoV-2 strains. As a first step, we assessed binding to variant receptor-binding domains (RBDs)

using a yeast display system (19) (Figure 1A). We incubated budding yeast displaying viral

RBDs on the cell surface with CDY14HL-Fc fusion protein and then assessed decoy binding via

112 flow cytometry by staining bound decoy with a fluorescent secondary antibody. CDY14HL-Fc

113 bound the ancestral (Wuhan-Hu1) RBD with an apparent affinity of $0.14 \mathrm{nM}$ (Figure 1B). This

114 result is in good agreement with the picomolar binding affinity we previously measured for the engineered decoy:RBD interaction using surface plasmon resonance (13).

117 Since our first description of CDY14HL (13), several SARS-CoV-2 variants of concern (VoCs)

118 have emerged with far greater transmissibility and clinical sequelae than the original Wuhan 119 strain (20); most of this evolution has occurred at the RBD:ACE2 interface (Figure 1C). We 120 used the yeast display system to evaluate decoy binding to five of these VoCs. We included an 121 additional RBD mutant not observed in natural SARS-CoV-2 isolates. This RBD sequence, 122 “Delta 4+," contains four additional substitutions (K417N, N439K, E484K, and N501Y) derived

123 from systematic analysis of RBD monoclonal antibody epitopes. These substitutions are

124 hypothesized to hold maximum potential for antibody escape (21). Remarkably, CDY14HL

125 maintained subnanomolar binding affinity for all VoC RBDs, including Iota, Delta +, Lambda, 126 and $\mathrm{Mu}$, and the "Delta 4+" RBD (Figure 1D). This finding is consistent with the broad 
127 resistance of CDY14HL to SARS-CoV-2 variant evolution previously observed in binding and

128 pseudotype neutralization studies (13).

129

130

131

132

133

134

135

136

137

138

139

140

141

142

143

144

145 
A

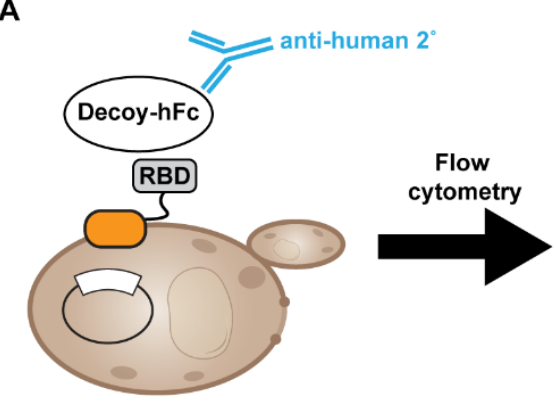

Budding yeast displaying RBD

C

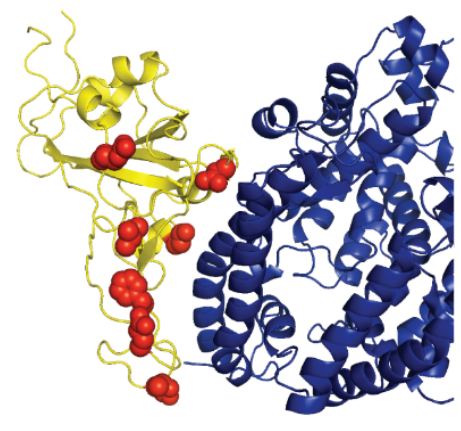

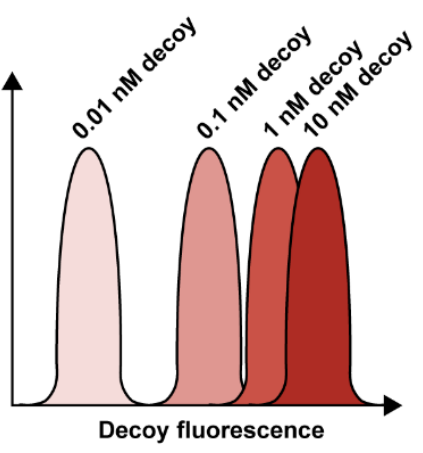

D
B

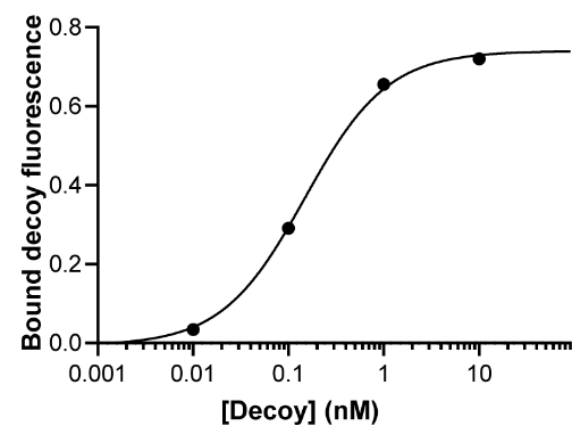

Fig 1. CDY14HL maintains tight binding to diverse SARS-CoV-2 variants

480
(A) Scheme for measuring decoy binding to yeast surface-expressed RBDs. (B) Representative decoy binding data for the RBD from the ancestral (Wuhan-Hu1) SARS-CoV-2 strain. (C) RBD amino acid positions mutated relative to the ancestral strain in the panel of SARS-CoV-2 variants are highlighted in red spheres on the yellow RBD ribbon diagram. These mutations cluster around the interface with ACE2 (blue ribbons), as shown by the coordinates of the complex (36) (D) Fitted values of the dissociation equilibrium constant $\left(K_{D}\right)$ for SARS-CoV-2 variants. 


\subsection{CDY14HL maintains potent neutralization for diverse SARS-CoV-2 variants}

147 We next investigated whether the broad decoy affinity for SARS-CoV-2 variants observed using

148 the yeast display binding assay would translate to potent viral neutralization. We first examined

149 the Omicron VoC. Unlike previous variants, which contain one, two, or three RBD mutations,

150 the Omicron RBD differs from the ancestral strain by 15 amino acids (Figure 2A) (22). This

151 level of mutation has caused a reduction in the efficacy of first-generation vaccines and most of

152 the monoclonal antibodies developed for therapeutic and passive prophylaxis applications (23-

153 28). We used a lentivirus harboring a luciferase reporter gene and pseudotyped with the SARS-

154 CoV-2 spike protein from the Omicron strain to measure the neutralization potency (half-

155 maximal inhibitory concentration [IC50]) of purified CDY14HL-Fc decoy (Figure 2B).

156 CDY14HL-Fc neutralizes Omicron more potently than the ancestral strain $(18 \mathrm{ng} / \mathrm{ml}$ vs. 35

$157 \mathrm{ng} / \mathrm{ml}$, Figure 2C). We extended this approach to include VoCs and variants of interest (VoI) not

158 previously evaluated. CDY14HL neutralized all SARS-CoV-2 strain pseudotypes tested,

159 including Lambda, Kappa, Delta, Delta +, Mu, and Zeta, with IC50 values near or below the

160 potency of the ancestral strain, Wuhan, against which it was engineered (Figure 2C). 
A

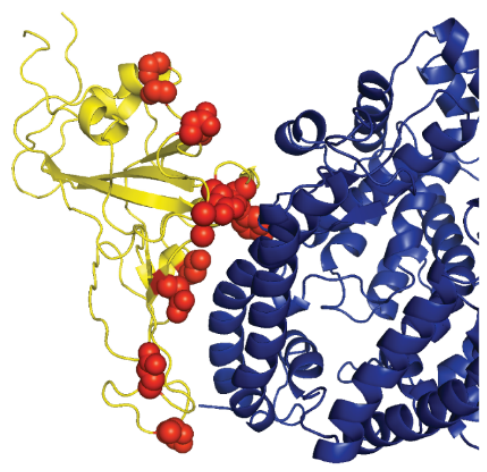

C

\begin{tabular}{|c|c|c|}
\hline RBD mutations & CoV-2 strain & $\begin{array}{c}\text { IC50 } \\
\text { (ng/ml) }\end{array}$ \\
\hline Reference & $\begin{array}{c}\text { ancestral } \\
\text { (Wuhan-Hu1) }\end{array}$ & 35 \\
\hline L452Q F490S & lambda & 11 \\
\hline L452R E484Q & kappa & 18 \\
\hline L452R T478K & delta & 14 \\
\hline K417N L452R T478K & delta plus & 10 \\
\hline $\begin{array}{c}\text { G339D S371L S373P S375F } \\
\text { K417N N440K G446S S477N } \\
\text { T478K E484A Q493K G496S } \\
\text { Q498R N501Y Y505H }\end{array}$ & omicron & 18 \\
\hline R346K E484K N501Y & mu & 21 \\
\hline E484K & zeta & 37 \\
\hline
\end{tabular}

B

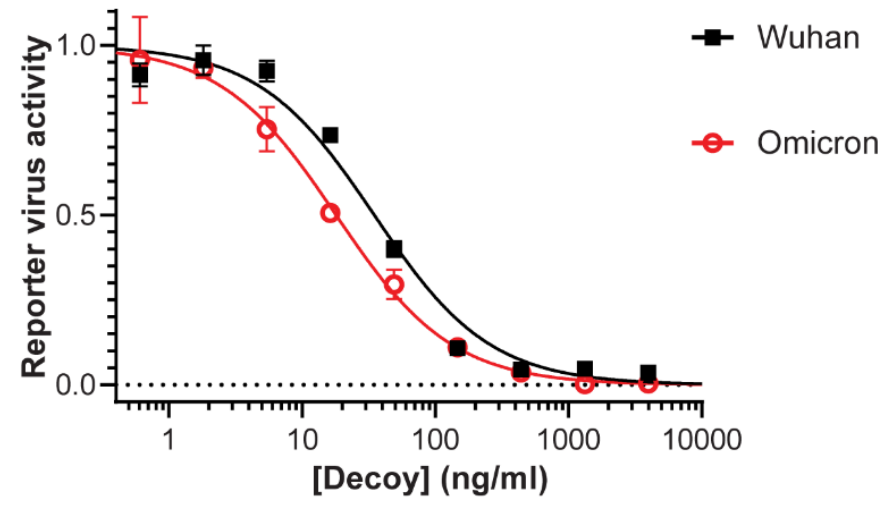

Fig 2. CDY14HL maintains potent neutralization for diverse SARS-CoV-2 variants

492 (A) RBD amino acid positions mutated relative to the ancestral strain in the Omicron variant of

493 SARS-CoV-2 are highlighted in red spheres on the yellow RBD ribbon diagram. These

494 mutations cluster around the interface with ACE2 (blue ribbons), as shown by the coordinates of

495 the complex(36). (B) Viral neutralization assay using lentiviruses pseudotyped with the ancestral

496 (Wuhan-Hu1) or Omicron variant spike protein. (C) Table of CDY14HL neutralization IC50

497 values collected for SARS-CoV-2 variant pseudotypes along with the RBD mutations of each

498 variant. 


\subsection{CDY14HL binds diverse ACE2-dependent CoVs}

168 Next, we evaluated the ability of CDY14HL to bind diverse RBDs from coronaviruses with

169 pandemic potential. We identified 10 sarbecoviruses isolated from bats in southern China and

$170 \operatorname{Laos}(12,29)$ that are thought to utilize ACE2 as a receptor (Figure 3A). We cloned synthetic

171 RBD genes into the yeast display format for binding analysis. Additionally, we included the

172 RBD from the human coronavirus NL63, an alpha-CoV with a genetically distinct RBD that has

173 been shown to use ACE2 for cell entry (30). We determined the binding affinities of yeast-

174 displayed RBD to CDY14HL-Fc by flow cytometry. Remarkably, the decoy bound to all but one

175 strain with subnanomolar affinity (Figure 3B). Research has recently shown that the weakest

176 decoy binder in the group, RATG13, also binds ACE2 more weakly than other members of the

177 clade (31). This behavior suggests that affinity for the decoy and the endogenous ACE2 receptor

178 are closely linked, as we have previously observed (13).

180 These binding data are in broad agreement with our previous work demonstrating tight decoy

181 binding to RBDs from SARS-CoV-1 and WIV1-CoV [(13). In the case of SARS-CoV-1, both

182 decoy and wild-type (wt) ACE2 (32) binding were weaker than for SARS-CoV-2, although the

183 decoy more potently neutralized SARS-CoV-1 than SARS-CoV-2 (18 ng/ml vs. $37 \mathrm{ng} / \mathrm{ml})(13)$.

184 Thus, decoy affinity measurements alone are not perfectly predictive of neutralizing potency.

185 Instead, the competitive binding of the viral spike for the decoy versus ACE2 receptors may

186 better predict neutralization. 
188 To assess this possibility, we employed a competitive binding assay between the decoy and

189 ACE2 receptor in the yeast system. We incubated RBD yeast with a low concentration of decoy

190 (1 nM of CDY14HL-hFc; $95 \mathrm{ng} / \mathrm{ml})$ along with a 100-fold molar excess of wt-ACE2 (100 nM of

191 wt-ACE2-mFc, with a mouse Fc fusion to distinguish it from the decoy). We assessed the level

192 of decoy binding retained in the presence of receptor competition by flow cytometry and

193 compared these values across the set of RBDs (Figure 3C). The positive control, the RBD from

194 the well-neutralized ancestral SARS-CoV-2 strain, retained $32 \%$ of decoy binding in the

195 presence of saturating levels of wt-ACE2-mFc (Figure 3D). Similar to SARS-CoV-2, all

196 sarbecovirus RBDs retained $>30 \%$ binding in the competition assay. Together with the observed

197 subnanomolar binding affinity, these data predict broad and potent neutralization of ACE2-

198 dependent beta-CoVs. The lone alpha-CoV in our study, NL63, retained a lower fraction of

199 decoy binding in the competition assay (23\%, Figure 3D). Further study is needed to determine

200 whether this result indicates a lower neutralizing potency of the decoy for the genetically distinct

201 ACE2-dependent alpha-CoVs. 
A

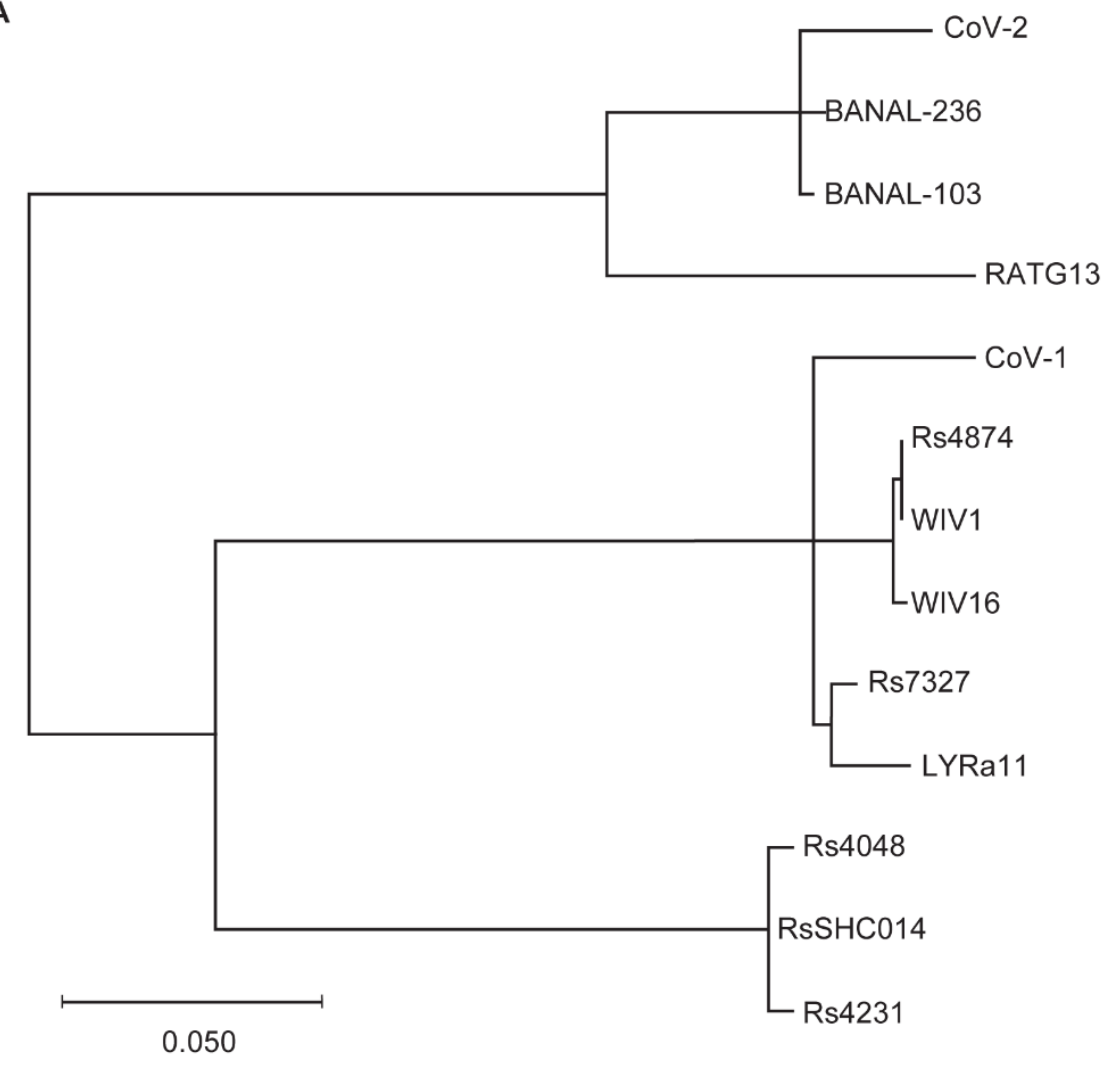

B

\begin{tabular}{|r|c|}
\hline \multicolumn{1}{|c|}{ Strain } & $\mathbf{K}_{\mathbf{D}}$ (nM) \\
\hline CoV-2 & 0.14 \\
\hline BANAL-103 & 0.38 \\
\hline BANAL-236 & 0.32 \\
\hline RATG13 & 1.24 \\
\hline RsSHC014 & 0.46 \\
\hline Rs4048 & 0.44 \\
\hline Rs4231 & 0.46 \\
\hline LYRa11 & 0.36 \\
\hline WIV16 & 0.27 \\
\hline Rs4874 & 0.26 \\
\hline Rs7327 & 0.33 \\
\hline NL63 & 0.36 \\
\hline &
\end{tabular}

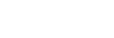

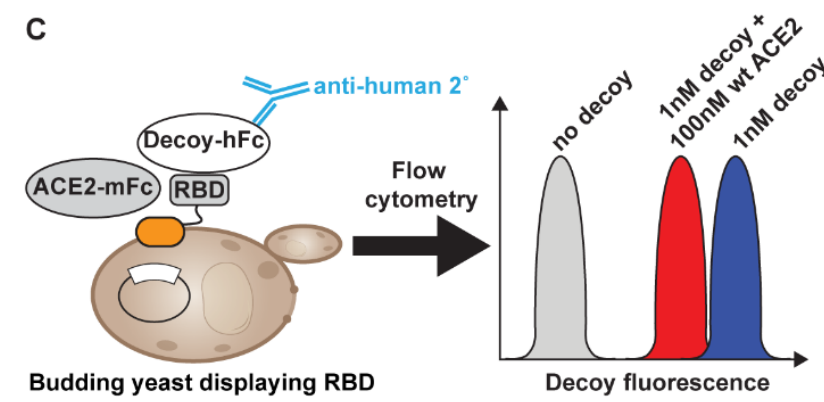

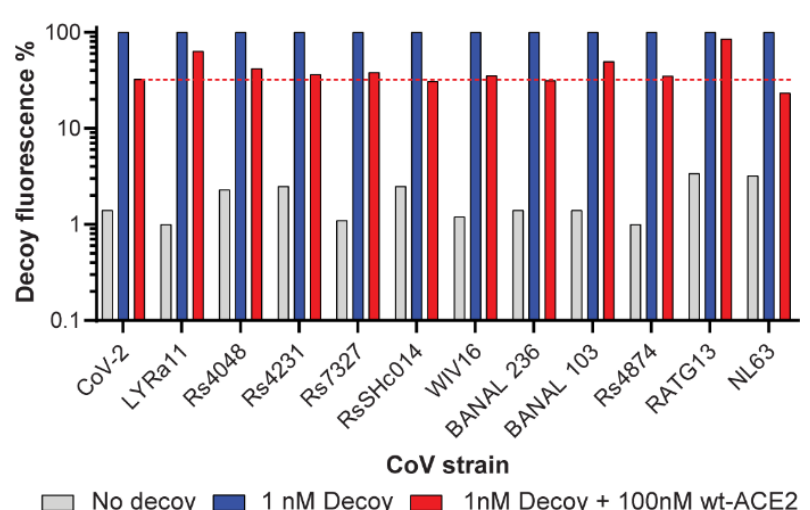

Fig 3. CDY14HL binds diverse ACE2-dependent CoVs

501 (A) Phylogenetic tree of sarbecovirus RBDs created using the maximum likelihood method. (B)

502 Table of dissociation equilibrium constants for the decoy interacting with various CoV RBDs.

503 (C) Schematic for measuring the competition between decoy and endogenous ACE2 receptor conditions, as assessed via the yeast display system. 


\section{Discussion}

210

211

212

213

214 following parenteral administration.

We previously reported the development of an affinity-matured, soluble ACE2 decoy, termed CDY14HL-Fc. This decoy binds and neutralizes SARS-CoV-2 strains from the early pandemic as well as the related pandemic sarbecovirus, SARS-CoV-1 (13). In this study, we have shown that the affinity-matured decoy retains broad neutralizing activity against every SARS-CoV-2 variant tested, including Delta, Delta + , and Omicron.

Our original strategy for deploying the decoy was developed in the context of preventing SARSCoV-2 infection. We accomplished this aim through the creation of an adeno-associated virus (AAV) vector expressing the decoy that is administered via nasal administration to engineer proximal airway cells to express neutralizing levels of the decoy at the airway surface (i.e., the virus' entry point). This approach could be particularly useful for immunocompromised patients who do not generate protective immunity following active vaccination. We are also developing the decoy as a therapeutic protein for treatment, or possibly prevention in high-risk groups,

225 The relentless emergence of new, highly transmissible SARS-CoV-2 variants in the current 226 pandemic reminds us of our vulnerability to the power of zoonosis and the intense selection 227 pressures experienced by pandemic viruses to evolve into more pathogenic and/or transmissible 228 variants. This experience suggests the importance of proactively developing countermeasures against future pandemics, which will likely be caused by a coronavirus based on the recent history of SARS, Middle East respiratory syndrome, and COVID-19. Indeed, CoVs constitute a 
231 major fraction of pre-zoonotic viruses ranked by multiple genetic and environmental factors for

232 pandemic potential (33). This threat compelled us to evaluate the competitive binding of our

233 ACE2 decoy to spike proteins from a variety of animal coronaviruses with zoonotic potential,

234 particularly sarbecoviruses that use ACE2 as a receptor. We were delighted to find that the

235 decoy retained very high binding activity against spike proteins from every pre-emergent strain

236 that was studied.

238 COVID-19 has illustrated how powerful the drive for viral fitness can be in circumventing

239 immunity generated from previous infection, vaccines, and antibody therapeutics. This rapid

240 evolution is substantially amplified in the setting of a global pandemic caused by a highly

241 transmissible virus. The use of a decoy protein based on a soluble version of a viral receptor

242 holds the promise of significantly restricting viral escape, as any mutation that diminishes decoy

243 binding will likely also diminish receptor binding and thus viral fitness. We are quickly moving

244 this ACE2 decoy into the clinic in the AAV platform as well as a protein therapeutic as a

245 possible solution to COVID-19 variants and to prepare for future coronavirus outbreaks. 


\section{Materials and Methods}

\subsection{CoV pseudotyped lentiviral neutralization assay}

254 Replication-incompetent lentiviruses pseudotyped with CoV spike proteins and packaging for a 255 Renilla luciferase reporter gene were purchased from Integral Molecular: RVP-701L Wuhan (lot 256 CL-114B), RVP-763L Delta (lot CL-267A), RVP-736L Zeta (lot CL-255A), RVP-730L Kappa 257 (lot CL-247A), RVP-768L Omicron (lot CL-297A), RVP-767L Mu (lot CL-274A), RVP-766L Lambda (lot CL-259A), and RVP-765L Delta + (lot CL-258A). We performed neutralization 259 assays using human embryonic kidney 293T cells overexpressing ACE2 (Integral Molecular) as 260 previously described (13).

\subsection{Recombinant protein production}

To generate wt-ACE2-Fc for competitive binding assays, we cloned human ACE2 (1-615) fused to a C-terminal mouse IgG2a Fc into pcDNA3.1. We transfected the plasmid into Expi293 cells and $150 \mathrm{mM} \mathrm{NaCl}$ buffer for purification on Protein A Sepharose 4B (ThermoFisher). The

267 protein was eluted in $0.1 \mathrm{M}$ citric acid, $\mathrm{pH} 3.0$ and neutralized in $1 \mathrm{M}$ Tris, $\mathrm{pH} 9.0$ before a final 268 buffer exchange to $25 \mathrm{mM}$ HEPES pH 7.2 and $150 \mathrm{mM} \mathrm{NaCl}$ by size-exclusion chromatography with Superose 6 resin (Cytiva). For these studies, we cloned the engineered CDY14HL 1-615

270 fragment in front of the human IgG1 Fc domain for expression and purification. We previously

271 characterized a decoy fusion to human IgG4 Fc (13), but found that Fc1 and Fc4 decoy fusions

272 behave similarly with respect to binding and neutralization (e.g., the IC50 values against Wuhan-

273 Hul pseudotypes were $37 \mathrm{ng} / \mathrm{ml}$ and $35 \mathrm{ng} / \mathrm{ml}$ for CDY14HL-Fc4 and CDY14HL-Fc1,

274 respectively). 


\subsection{Phylogenic tree construction}

277 The RBD sequences of the CoVs were taken from spike protein coding sequences downloaded

278 from the National Center for Biotechnology Information (NCBI). Using MEGA X (34), we

279 aligned the amino acid sequences in ClustalW and constructed a phylogenic tree using maximum

280 likelihood analysis.

281

282

\subsection{Yeast display binding assays}

283 The nucleic acid sequences of the CoV RBDs were taken from NCBI: CoV-2 (NC_045512.2),

(KY417151), RsSHC014 (KC881005), WIV16 (KT444582.1), BANAL-236 (MZ937003),

RBDs into a plasmid between an upstream Aga2 gene and a downstream hemagglutinin (HA) yeast with CDY14HL-Fc1 with or without wt-ACE-mFc for $30 \mathrm{~min}$ at $25^{\circ} \mathrm{C}$ before staining the sample with goat anti-human fluorescein isothiocyanate (FITC; ThermoFisher A18812) and

294 rabbit anti-HA-PE (Cell Signaling Technology 14904S). We used phosphate-buffered saline with

$2950.1 \%$ bovine serum albumin for all staining and washes. For the titration of CDY14HL-Fc1, we 296 incubated the yeast with 1:10 dilution series of CDY14HL-Fc1 at $25^{\circ} \mathrm{C}$ for $6 \mathrm{hr}$. The yeast were analyzed on an ACEA NovoCyte flow cytometer. We determined the level of CDY14HL-Fc1 
298 binding by taking the mean FITC signal for $500 \mathrm{RBD}+$ yeast cells collected for each condition.

299 We fitted the decoy concentration versus the decoy binding signal in GraphPad Prism using a

300 three-parameter fit to the binding isotherm.

301

302 Data Availability Statement

303 All data discussed in the manuscript are available in the main text.

304

305

306

307

308

309

310

311

312

313

314

315

316 


\section{Acknowledgments}

318 We thank Henry Hoff for protein expression and purification. We thank Nathan Denton for

319 assistance with manuscript preparation and graphics. 


\section{Author Contributions}

337 J.J.S. - conceptualization, data curation, formal analysis, investigation, methodology, project

338 administration, resources, supervision, validation, visualization, writing-original, writing-review,

339 and edits. S.L. - data curation, formal analysis, investigation, methodology, resources,

340 validation, visualization, writing-original, writing-review, and edits. J.M.W. - conceptualization,

341 funding acquisition, writing-original, writing-review, and edits.

342

343

344

345

346

347

348

349

350

351

352

353

354 
bioRxiv preprint doi: https://doi.org/10.1101/2022.01.17.476672; this version posted January 19, 2022. The copyright holder for this preprint (which was not certified by peer review) is the author/funder. All rights reserved. No reuse allowed without permission.

\section{$355 \quad$ Funding}

356 This work was funded by G2 Bio.

357

358

359

360

361

362

363

364

365

366

367

368

369

370

371

372 


\section{Conflict of Interest Statement}

374 J.M.W. is a paid advisor to and holds equity in Scout Bio and Passage Bio. He also holds equity

375 in the G2 Bio-associated asset companies and iECURE. He has sponsored research agreements

376 with Amicus Therapeutics, Biogen, Elaaj Bio, FA212, G2 Bio, G2 Bio-associated asset

377 companies, iECURE, Janssen, Passage Bio, and Scout Bio, which are licensees of Penn

378 technology. J.M.W. and J.J.S. are inventors on patents that have been licensed to various

379 biopharmaceutical companies and for which they may receive payments.

380

381

382

383

384

385

386

387

388

389

390

391 


\section{References}

1. Wec AZ, Wrapp D, Herbert AS, Maurer DP, Haslwanter D, Sakharkar M, et al. Broad neutralization of SARS-related viruses by human monoclonal antibodies. Science. 2020;369(6504):731-6. 2. Weinreich DM, Sivapalasingam S, Norton T, Ali S, Gao H, Bhore R, et al. REGN-COV2, a Neutralizing Antibody Cocktail, in Outpatients with Covid-19. N Engl J Med. 2021;384(3):238-51.

3. Weinreich DM, Sivapalasingam S, Norton T, Ali S, Gao H, Bhore R, et al. REGEN-COV Antibody Combination and Outcomes in Outpatients with Covid-19. N Engl J Med. 2021;385(23):e81.

4. O'Brien MP, Hou P, Weinreich DM. Subcutaneous REGEN-COV Antibody Combination to Prevent Covid-19. Reply. N Engl J Med. 2021;385(20):e70.

5. Starr TN, Greaney AJ, Addetia A, Hannon WW, Choudhary MC, Dingens AS, et al. Prospective mapping of viral mutations that escape antibodies used to treat COVID-19. Science. 2021;371(6531):850-4.

6. Barton MI, MacGowan SA, Kutuzov MA, Dushek O, Barton GJ, van der Merwe PA. Effects of common mutations in the SARS-CoV-2 Spike RBD and its ligand, the human ACE2 receptor on binding affinity and kinetics. Elife. 2021;10.

7. Van Egeren D, Novokhodko A, Stoddard M, Tran U, Zetter B, Rogers M, et al. Risk of rapid evolutionary escape from biomedical interventions targeting SARS-CoV-2 spike protein. PLoS One. 2021;16(4):e0250780.

8. Fenaux H, Gueneau R, Chaghouri A, Henry B, Mouna L, Roque-Afonso AM, et al. Emergence of SARS-CoV-2 resistance mutations in a patient who received anti-SARS-COV2 spike protein monoclonal antibodies: a case report. BMC Infect Dis. 2021;21(1):1223.

9. Jensen B, Luebke N, Feldt T, Keitel V, Brandenburger T, Kindgen-Milles D, et al. Emergence of the E484K mutation in SARS-COV-2-infected immunocompromised patients treated with bamlanivimab in Germany. Lancet Reg Health Eur. 2021;8:100164.

10. Rockett RJ, Basile K, Maddocks S, Fong W, Agius JE, Mackinnon JJ, et al. RESISTANCE CONFERRING MUTATIONS IN SARS-COV-2 DELTA FOLLOWING SOTROVIMAB INFUSION. medRxiv. 2021:2021.12.18.21267628.

11. Higuchi $Y$, Suzuki T, Arimori T, Ikemura N, Mihara E, Kirita $Y$, et al. Engineered ACE2 receptor therapy overcomes mutational escape of SARS-CoV-2. Nat Commun. 2021;12(1):3802.

12. Letko M, Marzi A, Munster V. Functional assessment of cell entry and receptor usage for SARSCoV-2 and other lineage B betacoronaviruses. Nat Microbiol. 2020;5(4):562-9.

13. Sims JJ, Greig JA, Michalson KT, Lian S, Martino RA, Meggersee R, et al. Intranasal gene therapy to prevent infection by SARS-CoV-2 variants. PLoS Pathog. 2021;17(7):e1009544.

14. Chan KK, Tan TJC, Narayanan KK, Procko E. An engineered decoy receptor for SARS-CoV-2 broadly binds protein $S$ sequence variants. Sci Adv. 2021;7(8).

15. Glasgow A, Glasgow J, Limonta D, Solomon P, Lui I, Zhang Y, et al. Engineered ACE2 receptor traps potently neutralize SARS-CoV-2. Proc Natl Acad Sci U S A. 2020;117(45):28046-55.

16. Havranek B, Chan KK, Wu A, Procko E, Islam SM. Computationally Designed ACE2 Decoy Receptor Binds SARS-CoV-2 Spike (S) Protein with Tight Nanomolar Affinity. J Chem Inf Model. 2021;61(9):4656-69.

17. Jing W, Procko E. ACE2-based decoy receptors for SARS coronavirus 2. Proteins. 2021;89(9):1065-78.

18. Chan KK, Dorosky D, Sharma P, Abbasi SA, Dye JM, Kranz DM, et al. Engineering human ACE2 to optimize binding to the spike protein of SARS coronavirus 2. Science. 2020;369(6508):1261-5.

19. Angelini A, Chen TF, de Picciotto S, Yang NJ, Tzeng A, Santos MS, et al. Protein Engineering and Selection Using Yeast Surface Display. Methods Mol Biol. 2015;1319:3-36. 
20. Campbell F, Archer B, Laurenson-Schafer H, Jinnai $\mathrm{Y}$, Konings F, Batra N, et al. Increased transmissibility and global spread of SARS-CoV-2 variants of concern as at June 2021. Euro Surveill.

$440 \quad 2021 ; 26(24)$.

21. Liu Y, Arase N, Kishikawa J-i, Hirose M, Li S, Tada A, et al. The SARS-CoV-2 Delta variant is poised to acquire complete resistance to wild-type spike vaccines. bioRxiv. 2021:2021.08.22.457114.

22. Miller NL, Clark T, Raman R, Sasisekharan R. Insights on the mutational landscape of the SARS-

23. Wilhelm A, Widera M, Grikscheit K, Toptan T, Schenk B, Pallas C, et al. Reduced Neutralization of SARS-CoV-2 Omicron Variant by Vaccine Sera and Monoclonal Antibodies. medRxiv.

$447 \quad 2021: 2021.12 .07 .21267432$.

448 24. Cao Y, Wang J, Jian F, Xiao T, Song W, Yisimayi A, et al. Omicron escapes the majority of existing SARS-CoV-2 neutralizing antibodies. Nature. 2021.

25. Cameroni E, Bowen JE, Rosen LE, Saliba C, Zepeda SK, Culap K, et al. Broadly neutralizing antibodies overcome SARS-CoV-2 Omicron antigenic shift. Nature. 2021.

26. Dejnirattisai W, Huo J, Zhou D, Zahradnik J, Supasa P, Liu C, et al. Omicron-B.1.1.529 leads to widespread escape from neutralizing antibody responses. bioRxiv. 2021.

27. VanBlargan L, Errico J, Halfmann P, Zost S, Crowe J, Purcell L, et al. An infectious SARS-CoV-2 B.1.1.529 Omicron virus escapes neutralization by therapeutic monoclonal antibodies. Res Sq. 2021. 28. Dejnirattisai W, Shaw RH, Supasa P, Liu C, Stuart AS, Pollard AJ, et al. Reduced neutralisation of SARS-CoV-2 omicron B.1.1.529 variant by post-immunisation serum. Lancet. 2022;399(10321):234-6. 29. Islam A, Ferdous J, Sayeed MA, Islam S, Kaisar Rahman M, Abedin J, et al. Spatial epidemiology and genetic diversity of SARS-CoV-2 and related coronaviruses in domestic and wild animals. PLoS One. 2021;16(12):e0260635.

461 30. Hofmann H, Pyrc K, van der Hoek L, Geier M, Berkhout B, PohImann S. Human coronavirus NL63

462 employs the severe acute respiratory syndrome coronavirus receptor for cellular entry. Proc Natl Acad Sci U S A. 2005;102(22):7988-93.

464 31. Wrobel AG, Benton DJ, Xu P, Roustan C, Martin SR, Rosenthal PB, et al. Author Correction: SARSCoV-2 and bat RaTG13 spike glycoprotein structures inform on virus evolution and furin-cleavage effects. Nat Struct Mol Biol. 2020;27(10):1001.

467 32. Walls AC, Park YJ, Tortorici MA, Wall A, McGuire AT, Veesler D. Structure, Function, and 468 Antigenicity of the SARS-CoV-2 Spike Glycoprotein. Cell. 2020;181(2):281-92 e6.

469 33. Grange ZL, Goldstein T, Johnson CK, Anthony S, Gilardi K, Daszak P, et al. Ranking the risk of 470 animal-to-human spillover for newly discovered viruses. Proc Natl Acad Sci U S A. 2021;118(15).

471 34. Kumar S, Stecher G, Li M, Knyaz C, Tamura K. MEGA X: Molecular Evolutionary Genetics Analysis 472 across Computing Platforms. Mol Biol Evol. 2018;35(6):1547-9.

$473 \quad 35 . \quad$ Chao G, Lau WL, Hackel BJ, Sazinsky SL, Lippow SM, Wittrup KD. Isolating and engineering 474 human antibodies using yeast surface display. Nat Protoc. 2006;1(2):755-68.

475 36. Xu C, Wang Y, Liu C, Zhang C, Han W, Hong X, et al. Conformational dynamics of SARS-CoV-2 476 trimeric spike glycoprotein in complex with receptor ACE2 revealed by cryo-EM. Sci Adv. 2021;7(1). 